\title{
Resistance to endoplasmic reticulum stress is an acquired cellular characteristic of rheumatoid synovial cells
}

\author{
SATOSHI YAMASAKI ${ }^{1}$, NAOKO YAGISHITA ${ }^{1}$, KANEYUKI TSUCHIMOCHI $^{1,4}$, YUKIHIRO KATO ${ }^{1}$, \\ TAKESHI SASAKI ${ }^{1}$, TETSUYA AMANO ${ }^{1}$, MOROE BEPPU ${ }^{3}$, HARUHITO AOKI ${ }^{3}$, \\ HIROSHI NAKAMURA ${ }^{2}$, KUSUKI NISHIOKA ${ }^{2}$ and TOSHIHIRO NAKAJIMA ${ }^{1}$ \\ Departments of ${ }^{1}$ Genome Science, and ${ }^{2}$ Rheumatology, Immunology and Genetics Program, Institute of \\ Medical Science, ${ }^{3}$ Department of Orthopaedic Surgery, St. Marianna University School of Medicine, \\ 2-16-1 Sugao, Miyamae-ku, Kawasaki, Kanagawa 216-8512; ${ }^{4}$ Department of Orthopaedic Surgery, \\ Kagoshima Graduate School of Medical and Dental Sciences, Kagoshima 890-8520, Japan
}

Received January 10, 2006; Accepted February 24, 2006

\begin{abstract}
Synoviolin is an endoplasmic reticulum (ER)resident E3 ubiquitin ligase which plays a critical role in ERassociated degradation (ERAD). We found that Synoviolin is a novel causative factor for rheumatoid arthritis (RA), which is especially up-regulated in proliferating synovial cells in the disease. We attempted to examine the role of Synoviolin in ER stress-induced apoptosis and proliferation of synovial cells. RA synovial cells (RSCs) were refractory to ER stressinduced apoptosis compared with HEK293 or HeLa cells. RSCs were also more resistant to the apoptosis than synovial cells from osteoarthritis patients, significantly. Down-regulation of Synoviolin by siRNA increased the susceptibility to ER stress-induced apoptosis in RSCs. Knock-down of Synoviolin by siRNA did not only induce apoptosis of RSCs but also inhibited their proliferation in vitro. These data suggest that RSCs are extraordinarily refractory to ER stress-induced apoptosis, and we termed this special property 'hyperERAD'. Since Synoviolin is overexpressed in RSCs, and is known to play a critical role in the ERAD system as E3 ubiquitin ligase, hyper-ERAD is likely to present in these cells. Subsequently, the hyper-ERAD may cause synovial hyperplasia through its anti-apoptotic effect in RA. Further analyses are necessary to address this point, however, resistance to ER stress-induced apoptosis, or hyper-ERAD is a noteworthy new cellular characteristic of RSCs.
\end{abstract}

Correspondence to: Dr Toshihiro Nakajima, Department of Genome Science, Institute of Medical Science, St. Marianna University School of Medicine, 2-16-1 Sugao, Miyamae-ku, Kawasaki, Kanagawa 216-8512, Japan

E-mail: nakashit@marianna-u.ac.jp

Key words: Synoviolin, Hrd1, endoplasmic reticulum-associated degradation, apoptosis, proliferation

\section{Introduction}

Rheumatoid arthritis (RA) is characterized by abnormal immunity and synovial fibroblasts overgrowth followed by bone and cartilage destruction. While the immunological aspects of RA are well understood, the molecular basis for promoting overgrowth of rheumatoid synovial cells (RSCs) remains elusive $(1,2)$. We recently succeeded in identifying Synoviolin as a possible pathogenic factor for RA (3). Synoviolin is an endoplasmic reticulum (ER)-resident ubiquitin ligase with $\mathrm{N}$-terminal six transmembrane domains and RING finger motif $(4,5)$. Synoviolin is involved in ER-associated degradation (ERAD), which is essential for maintenance of ER function, and activation of ERAD is important to eliminate proteins with an incorrect three-dimensional structure (unfolded protein) through the ubiquitin and proteasome system (3-6). The importance of Synoviolin in the ERAD system is clearly confirmed by this gene being embryonically lethal in the phenotypes of homozygous knock-out mice $(3,7)$.

Both 'loss of function' and 'gain of function' using transgenic technology, Synoviolin is proved to be a pathogenic factor for arthropathy at least in mice (3). Detailed examination of synovial tissue from synoviolin heterozygous knock-out mice $\left(\right.$ syno $^{+/-}$) with collagen-induced arthritis (CIA) revealed that there is an aberrantly increased apoptosis of synovial cells, which inhibited the progression of synovial hyperplasia in the mice, in spite of comparable productions of anti-type II collagen antibodies, tumor necrosis factor $\alpha$ $(\mathrm{TNF} \alpha$ ), and interleukin 1 (IL-1) to those of wild-type mice. Conversely, there was remarkable synovial cell overgrowth in Synoviolin overexpressing mice. These results indicate that Synoviolin accelerates synovial cell overgrowth, and consequently leads to arthropathy in mice. Synoviolin overexpressing mice can be regarded as a novel RA model that firstly demonstrates the possible importance of hyperactivation of the ERAD system (hyper-ERAD) in overgrowth of RSCs, because RSCs express high levels of Synoviolin protein (3). To address this hypothesis, we examined whether RSCs are equipped with the hyper-ERAD system by Synoviolin, and 
A

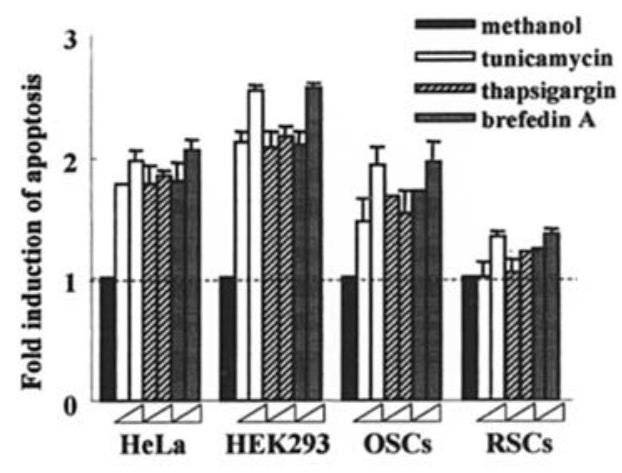

B

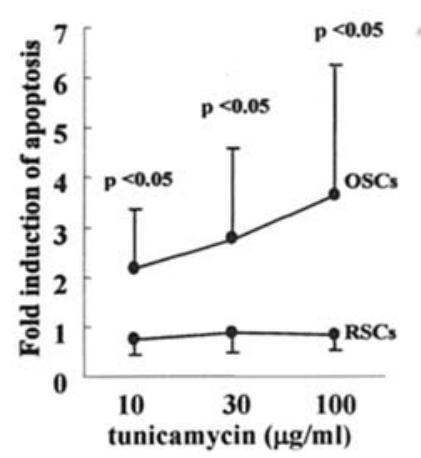

Figure 1. Rheumatoid synovial cells are refractory to ER stress-induced apoptosis. (A) HeLa, HEK293, and RSCs or OA synovial cells (OSCs) were treated with tunicamycin $(10$ or $100 \mu \mathrm{g} / \mathrm{ml})$, thapsigargin $(1$ or $10 \mu \mathrm{M})$, breferdin A (10 or $100 \mu \mathrm{g} / \mathrm{ml}$ ) or methanol (negative control) for $48 \mathrm{~h}$. Apoptosis was evaluated using an ssDNA apoptosis ELISA kit. (B) RSCs $(n=5)$ and OSCs $(n=5)$ were treated with tunicamycin for $48 \mathrm{~h}$. Apoptosis was evaluated by ssDNA Apoptosis ELISA kit.

how hyper-ERAD affects the proliferation and apoptosis of RSCs in this study.

\section{Materials and methods}

Reagents. ER stress inducers, including tunicamycin, thapsigargin and breferdin A, were obtained from Sigma (St. Louis, $\mathrm{MO})$ to induce ER stress in various cells (8).

Synovial cell isolation and culture. Synovial cells were isolated from synovial tissue obtained from 10 patients with rheumatoid arthritis (RA) who met the American College of Rheumatology criteria for RA at the time of orthopedic surgery (9). In some experiments, synovial cells isolated from patients with osteoarthritis (OA) were also used. These cells were cultured in DMEM containing $1 \%$ penicillin, $1 \%$ streptomycin and $10 \%$ fetal bovine serum. The adherent synovial cells used in this study at third to fifth passages were $<1 \%$ reactive with monoclonal antibodies, including CD3, CD68, CD20, and von Willebrand factor.

RNA interference assay. Small interfering RNA (siRNA) with 21 nucleotides was chemically synthesized (Hokkaido System Science Co., Japan). The siRNA for synoviolin was created by annealing 5'-CGU UCC UGG UAC GCC GUC ATT-3' (sense sequence) and 5'-UGA CGG CGU ACC AGG AAC GTT-3' (anti-sense). The sense sequence corresponds to the sequence of synoviolin cDNA (238-256), and the TT of $3^{\prime}$ end is overhanging nucleotides. Transfection was carried out by using Oligofectamine (Invitrogen, CA) at the concentration of $20 \mathrm{nM}$ annealed RNA duplex, according to the instructions provided by the supplier. The siRNA for green fluorescent protein (GFP) was used as negative control.

Western blot analysis. Cell cultures were harvested and lysed in buffer containing $25 \mathrm{mM}$ Tris- $\mathrm{HCl}(\mathrm{pH} 6.8), 50 \mathrm{mM} \mathrm{NaCl}$, $1 \%$ NP-40, $0.25 \%$ SDS and protease inhibitors, and aliquots of clear cell lysates were separated on SDS-polyacrylamide gels, transferred onto nitrocellulose membrane, and immunoblotted with anti-Synoviolin monoclonal antibodies (Abs) (3). Bound antibodies were detected by peroxidase-conjugated goat anti-mouse Abs and ECL detection system (Amersham Pharmacia Biotech).

Apoptosis assay. Apoptosis of RSCs was determined using an ssDNA apoptosis ELISA kit (Chemicon International, Inc., CA). ELISA was carried out according to the manufacturer's protocol. The extent of apoptosis was evaluated as a fold induction compared with control cells. In some experiments, apoptosis was detected by staining cells with propidium iodide and analyzing them for DNA content on a FACScan to detect hypodiploid DNA.

Proliferation assay. The proliferation of RSCs was evaluated using Cell Counting Kit-8 (Dojindo, Kumamoto, Japan) according to the instructions provided by the manufacturer.

Statistical analysis. Data are expressed as mean \pm SD. Differences between control and treated cells were examined for statistical significance using the Student's t-test. A Pvalue $<0.05$ denoted the presence of a statistically significant difference.

Ethical considerations. All human experimental protocols described in this study were approved by the Ethics Review Committees of St. Marianna University School of Medicine. Before obtaining human tissue, a signed consent form was obtained from each subject participating in the study.

\section{Results}

RSCs are refractory to ER stress-induced apoptosis. First, we simply questioned whether RSCs are refractory to apoptosis induced by excess accumulation of unfolded proteins in ER. RSCs are expected to have high ability to eliminate these unfolded proteins from ER through the 'hyper-ERAD' system by their high expression levels of Synoviolin. To address this issue, pharmacological analysis was performed by using ER stress inducers to evaluate the ERAD function of RSCs. We compared the sensitivities of RSCs to ER stress inducers with that of OA synovial cells (OSCs), human embryonic kidney (HEK293) cell, or HeLa cells, which are known to express a lower amount of Synoviolin (3; data not shown). These cells were treated with ER stress-inducing reagents (tunicamycin, thapsigargin and breferdin A) to load unfolded proteins in ER, and the susceptibility of these cells to ER stress-induced apoptosis was determined by ELISA. As expected, RSCs 
$\mathbf{A}$

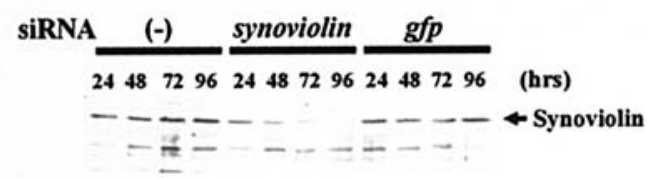

B

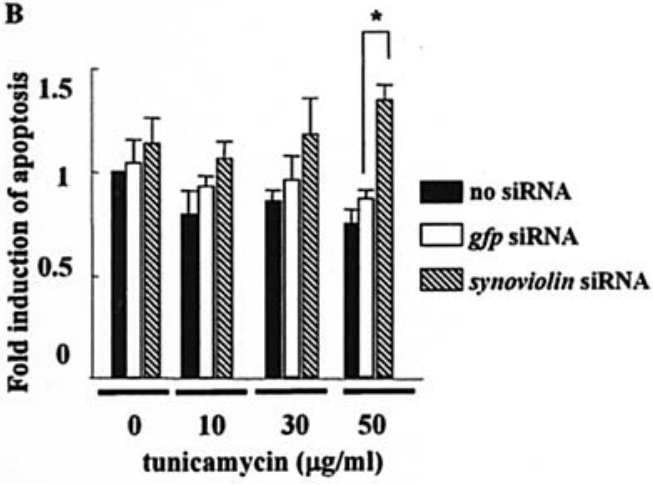

Figure 2. Synoviolin siRNA increase the sensitivity to ER stress-induced apoptosis. (A) Down-regulation of Synoviolin in RSCs by siRNA. (B) RSCs were treated with siRNA for $72 \mathrm{~h}$, and the cells were further treated with an indicated dose of tunicamycin for $48 \mathrm{~h}$. Apoptosis was evaluated by using ELISA. ${ }^{*} \mathrm{P}<0.05$.

A

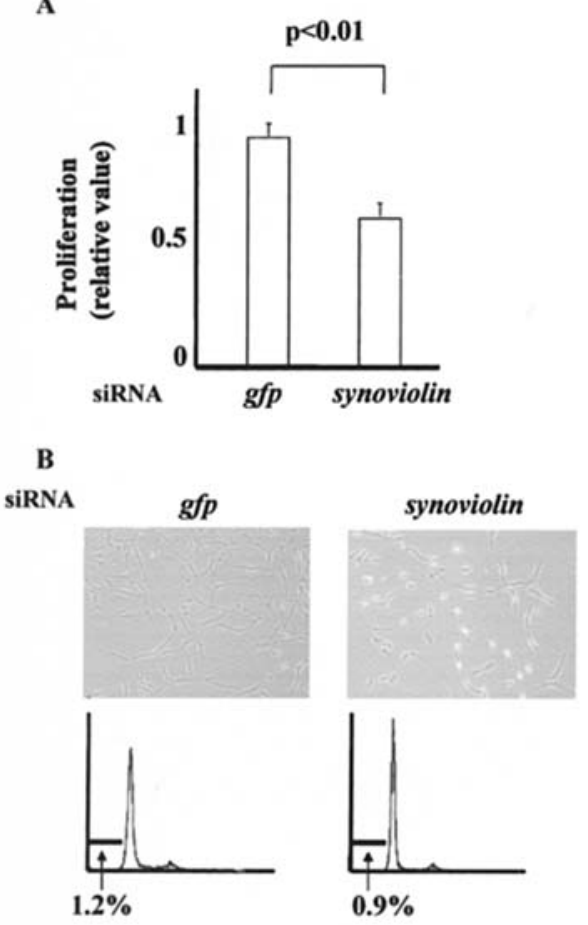

Figure 3. Down-regulation of Synoviolin inhibits RSC proliferation. (A) RSCs were treated with siRNA for $96 \mathrm{~h}$, and the proliferation of the cells was evaluated using Cell Counting kit-8. (B) Morphological change (upper) and apoptosis assay (lower) of RSCs treated with siRNA for Synoviolin for $96 \mathrm{~h}$. RSCs were treated with siRNA for $96 \mathrm{~h}$, and the apoptosis of the cells was detected by FACScan after propidium iodide staining.

were found to be remarkably refractory to ER stress-induced apoptosis compared with OSCs, HEK293 cells or HeLa cells (Fig. 1A). Since RSCs were apparently more resistant to ER stress-induced apoptosis than OSCs, we further evaluated whether RSCs are more resistant to ER stress, and found that RSCs were more refractory to ER stress-induced apoptosis than OSCs, significantly (Fig. 1B; $10 \mu \mathrm{g} / \mathrm{ml}$ : RSCs $0.73 \pm 0.30$, OSCs $2.18 \pm 1.19 ; 30 \mu \mathrm{g} / \mathrm{ml}$ : RSCs $0.84 \pm 0.35$, OSCs $2.76 \pm 1.84 ; 100 \mu \mathrm{g} / \mathrm{ml}$ : RSCs $0.81 \pm 0.28$, OSCs $3.65 \pm 2.53)$. It should be noted that apoptosis of RSCs was not induced at a considerably high concentration of tunicamycin (8). These data clearly indicate that RSCs are distinctly equipped with a high ability to deal with a large amount of unfolded proteins in ER.

Down-regulation of Synoviolin promotes ER stress-induced apoptosis of RSCs. Next, we addressed whether 'hyper-ERAD' is achieved by high expression of Synoviolin in RSCs. We administered an indicated dose of tunicamycin to RSCs after the down-regulation of Synoviolin expression by 72-h treatment with siRNA for Synoviolin. Treatment of RSCs with siRNA for Synoviolin efficiently suppressed the protein expression of Synoviolin (Fig. 2A). The siRNA for Synoviolin alone did not enhance the apoptosis of RSCs (Fig. 2B). Treatment with tunicamycin did not induce significant apoptosis again in RSCs treated with control siRNA. However, synoviolin knock-down significantly enhanced the apoptosis of RSCs induced by tunicamycin at a concentration of $50 \mu \mathrm{g} /$ $\mathrm{ml}$ compared with those treated with control siRNA, even though the sensitivity of these cells to apoptosis is still very low compared with that of other cells (Fig. 2B). This data indicated that RSCs restored the sensitivity to ER stressinduced apoptosis by Synoviolin knock-down. Thus, it can be said that Synoviolin is important, at least in part, for the hyper-ERAD of RSCs, and for RSC survival in ER stress milieu.

Down-regulation of Synoviolin inhibits proliferation of RSCs. One of the most striking data of our previous report about Synoviolin is that Synovioin overexpressing mice develop arthropathy with synovial cell overgrowth. These results strongly suggest that Synoviolin is also required for synovial cell overgrowth. To verify the importance of Synoviolin in RSC proliferation, we carried out more detailed examinations of the proliferation of Synoviolin down-regulated RSCs. As we expected, the proliferation of RSCs was significantly inhibited by $96 \mathrm{~h}$ after treatment with siRNA for Synoviolin compared with RSCs treated with siRNA for GFP (Fig. 3A). Since the appearance of RSCs treated with siRNA for Synoviolin by phase contrast microscopic examination shows morphological change (Fig. 3B), we examined whether there are apoptotic cells in them by flow cytometry. There was no increment of hypodiproid DNA in Synoviolin down-regulated RSCs by 96-h treatment with siRNA for Synoviolin (Fig. 3B). These results are consistant with the data shown in Fig. 2B, and indicate that down-regulation of Synoviolin inhibits the proliferation of RSCs without inducing apoptosis of them.

\section{Discussion}

Based on our serial experiments, we propose 'hyper-ERAD' as a novel pathogenic mechanism that promotes RSC survival and proliferation, and that this machinery can be mediated by up-regulation of Synoviolin in RA. RSCs, equipped with 
'hyper-ERAD', are proved to be a unique cell with high ability to accommodate severe ER stress (Fig. 1). Downregulation of Synoviolin resulted in aberrant apoptosis of synovial cells in CIA-induced syno ${ }^{+/}$mice (3). Likewise, down-regulation of the gene in RSCs resulted in increased apoptosis induced by ER stress inducers (Fig. 2). These accumulating data strongly support our novel disease concept that synovial cell overgrowth is triggered, at least in part, by hyper-ERAD, which consequently leads to synovial hyperplasia in RA. The importance of Synoviolin in 'hyper-ERAD' is also confirmed in this study.

Another remarkable aspect of Synoviolin is its ability to regulate the proliferation of synovial cells both in vivo and in vitro. Synoviolin overexpressing mice show spontaneous overgrowth of synovial cells (3), and siRNA for Synoviolin abrogates the proliferation of RSCs in vitro (Fig. 3). In addition, our recent analyses of the synoviolin promoter also demonstrated that transcriptional regulation of the gene is critical for cell growth and apoptosis in vitro (10), which strongly suggests that the amount of Synoviolin is critical to the regulation of synovial cell overgrowth.

Further examination is required to understand how Synoviolin regulates the proliferation of RSCs on a molecular basis. First, it is necessary to determine the alternation of unfolded protein response (UPR) by Synoviolin. It is believed that dual response UPR and the ERAD are triggered by the ER dysfunction or so-called 'ER stress', and both responses cooperate to maintain ER homeostasis $(6,11)$. The ER-resident kinase, PKR-like ER kinase (PERK), is known to be activated as one UPR response. PERK phosphorylates the eukaryotic initiation factor $(\mathrm{eIF} 2 \alpha)$ that attenuates global translation to block the influx of newly synthesized proteins into ER (12). Therefore, protein synthesis for cell proliferation becomes insufficient if the ERAD system does not work sufficiently enough to avoid ER stress and eventually triggers UPR. GADD153 is another candidate molecule that explains the importance of Synoviolin in RSC proliferation because it is thought to be activated in cells with ER stress, and causes cell cycle arrest (13). Actually, cell cycle study by staining cells with propidium iodide demonstrates that the cell treated with siRNA for Synoviolin seems to be arrested at G1 or G0 phase (Fig. 3B). Further detailed studies are necessary to clarify the whole mechanism of UPR activation in RSCs with Synoviolin down-regulation.

Is 'hyper-ERAD' really necessary for overgrowth of RSCs? It is easy to assume that 'hyper-ERAD' is a very favourable function for RSCs because the articular milieu in inflamed joints of RA is extremely hostile to ER. It is not difficult to find the cause of ER stress in inflamed joints of RA. For example, hypoxia frequently occurs in inflamed joints of RA, which is known to inhibit ER function (12). In addition, proinflammatory cytokines promote the synthesis of various proteins, including hyaluronate, cytokines and proteases in RSCs, which inevitably accelerates the accumulation of unfolded protein in ER. Indeed, nuclear translocation of ATF6, an ER-resident transcriptional factor that translocates to the nucleus under ER stress conditions (14), was certainly confirmed in RA synovia (data not shown). A report from another laboratory also implicates the existence of ER stress by demonstrating the high expression of $\mathrm{BiP}$, a well-known transcriptional target for ATF6 (15). Thus, it is rational that 'hyper-ERAD' evoked by Synoviolin overexpression in RSCs helps RSCs to prevent these extra- and/or intra-cellular environmental stresses and subsequent apoptosis.

Here, we propose a concept that hyper-ERAD is a novel pathogenic factor for RA, which is especially important in RSC overgrowth. Historically, the autonomous proliferation of RSCs is thought to be due to high expression of protooncogenes, such as $c$-myc, fos, ras, or virus transforming gene tax or activation of specific transcriptional factors, such as $\mathrm{NF}-\mathrm{\kappa B}(16,17)$. Synoviolin is also a member of the genes responsible for synovial cell overgrowth. Moreover, Synoviolin is one of the most convincing molecules for the RA therapy targeting RSCs because it is an enzyme and enzymes are regarded as the most appropriate target for drug development. The detailed molecular mechanisms are still unclear and various approaches are required to prove our hypothesis; however, it should be noted that resistance to ER stressinduced apoptosis is a novel cellular characteristic of RSCs.

\section{Acknowledgements}

We thank Drs T. Ohta and K. Tanaka for the critical comments. We are grateful to S. Toriyama, N. Furuya, H. Takahashi, N. Takagi, M. Hinata, S. Shinkawa, A. Sugamiya, and Y. Nakagawa for the excellent technical assistance. This study was supported by LocomoGene Inc. and grants from Ministry of Education, Culture, Sports, Science and Technology, Japan Society for the Promotion of Science, Regional Science Promotion Program in Kanagawa, The Kanagawa HighTechnology Foundation, Kanagawa Academy of Science and Technology Research Grants, Mochida Pharmaceutical Co., Ltd., Pharmaceuticals and Medical Devices Agency, and New Energy and Industrial Technology development organization.

\section{References}

1. Pap T, Muller-Ladner U, Gay RE and Gay S: Fibroblast biology. Role of synovial fibroblasts in the pathogenesis of rheumatoid arthritis. Arthritis Res 2: 361-367, 2000.

2. Szekanecz Z and Koch AE: Update on synovitis. Curr Rheumatol Rep 3: 53-63, 2001.

3. Amano T, Yamasaki S, Yagishita N, et al: Synoviolin/Hrd1, an E3 ubiquitin ligase, as a novel pathogenic factor for arthropathy. Genes Dev 17: 2436-2449, 2003.

4. Kaneko M, Ishiguro M, Niinuma Y, Uesugi M and Nomura Y: Human HRD1 protects against ER stress-induced apoptosis through ER-associated degradation. FEBS Lett 532: 147-152, 2002.

5. Bays NW, Gardner RG, Seelig LP, Joazeiro CA and Hampton RY: Hrd1p/Der3p is a membrane-anchored ubiquitin ligase required for ER-associated degradation. Nat Cell Biol 3: 24-29, 2001.

6. Kaufman RJ: Orchestrating the unfolded protein response in health and disease. J Clin Invest 110: 1389-1398, 2002.

7. Yagishita N, Ohneda K, Amano T, et al: Essential role of synoviolin in embryogenesis. J Biol Chem 280: 7909-7916, 2005.

8. Chae $\mathrm{HJ}, \mathrm{Kim} \mathrm{HR}, \mathrm{Xu} \mathrm{C}$, et al: $\mathrm{BI}-1$ regulates an apoptosis pathway linked to endoplasmic reticulum stress. Mol Cell 15: 355-366, 2004.

9. Arnett FC, Edworthy SM, Bloch DA, et al: The American Rheumatism Association 1987 revised criteria for the classification of rheumatoid arthritis. Arthritis Rheum 31: 315-324, 1988.

10. Tsuchimochi K, Yagishita N, Yamasaki S, et al: Identification of a crucial site for synoviolin expression. Mol Cell Biol 16: 7344-7356, 2005.

11. Friedlander R, Jarosch E, Urban J, Volkwein C and Sommer T: A regulatory link between ER-associated protein degradation and the unfolded-protein response. Nat Cell Biol 2: 379-384, 2000. 
12. Koumenis C, Naczki C, Koritzinsky M, et al: Regulation of protein synthesis by hypoxia via activation of the endoplasmic reticulum kinase PERK and phosphorylation of the translation initiation factor eIF2alpha. Mol Cell Biol 22: 7405-7416, 2002.

13. Barone MV, Crozat A, Tabaee A, Philipson L and Ron D: CHOP (GADD153) and its oncogenic variant, TLS-CHOP, have opposing effects on the induction of G1/S arrest. Genes Dev 8: 453-464, 1994.

14. Shuda M, Kondoh N, Imazeki N, et al: Activation of the ATF6, XBP1 and grp78 genes in human hepatocellular carcinoma: a possible involvement of the ER stress pathway in hepatocarcinogenesis. J Hepatol 38: 605-614, 2003.
15. Blass $\mathrm{S}$, Union A, Raymackers $\mathrm{J}$, et al: The stress protein $\mathrm{BiP}$ is overexpressed and is a major B and T cell target in rheumatoid arthritis. Arthritis Rheum 44: 761-771, 2001.

16. Qu Z, Garcia CH, O'Rourke LM, Planck SR, Kohli M and Rosenbaum JT: Local proliferation of fibroblast-like synoviocytes contributes to synovial hyperplasia. Results of proliferating cell nuclear antigen/cyclin, c-myc, and nucleolar organizer region staining. Arthritis Rheum 37: 212-220, 1994.

17. Nakajima T, Aono H, Hasunuma T, et al: Overgrowth of human synovial cells driven by the human $\mathrm{T}$ cell leukemia virus type I tax gene. J Clin Invest 92: 186-193, 1993. 